Voix et Images

\title{
La Chair de pierre de Jacques Folch-Ribas : un roman exceptionnel
}

\section{Pierre Hébert}

Volume 16, numéro 1 (46), automne 1990

Les correspondants littéraires d'Alfred DesRochers

URI : https://id.erudit.org/iderudit/200886ar

DOI : https://doi.org/10.7202/200886ar

Aller au sommaire du numéro

Éditeur(s)

Université du Québec à Montréal

ISSN

0318-9201 (imprimé)

1705-933X (numérique)

Découvrir la revue

Citer cet article

Hébert, P. (1990). La Chair de pierre de Jacques Folch-Ribas : un roman exceptionnel. Voix et Images, 16(1), 166-169. https://doi.org/10.7202/200886ar d'utilisation que vous pouvez consulter en ligne.

https://apropos.erudit.org/fr/usagers/politique-dutilisation/ 


\section{Roman}

\section{La Chair de pierre de Jacques Folch-Ribas: un roman exceptionnel}

\section{par Pierre Hébert, Université de Sherbrooke}

Un bourg parisien, vers le milieu du XVII ${ }^{e}$ siècle. Claude Baillif regarde des artisans dresser une charpente. Il reste là pendant des heures, sans manger, fasciné par la géométrie de l'édifice que des ouvriers construisent: C'était un mystère de religion, une mécanique inconnue, une jonglerie qui faisait tenir ensemble les cheurons bien droits, les uns à côté des autres [...]. ${ }^{1}$

Or, comme son personnage principal Claude Baillif, je contemple, moi aussi, ce merveilleux roman qu'est la Chair de pierre, de Jacques Folch-Ribas, cet édifice superbe, monument exceptionnel à l'architecture, à la beauté, à l'humilité également.

La Chair de pierre raconte en effet la vie de Claude Baillif, premier architecte de la Nouvelle-France, né en France vers 1635, et décédé en Nouvelle-France vers 1698. Sur les origines familiales, le récit se montre discret: Claude Baillif $a$ onze ans lorsque son père, constructeur de moulins à vent, meurt au moment où toute la famille fuyait son petit village de Normandie parce que le plus jeune des frères avait contracté la peste. Quelque temps après, le jeune Claude rencontre une fillette dont il s'éprendra, et qui d'ailleurs hantera le reste de ses jours: Catherine. Ainsi s'ouvre la Chair de pierre, sous les signes liés de la mort et de l'amour.

Toutefois, Baillif rencontrera inopinément Maître Nicolas de Limbourg, architecte. Apprenant que Baillif est fils d'un charpentier boulangeur, Nicolas de Limbourg reconnaît en lui les qualités de l'architecte, et il décide alors de l'initier à une religion dont les prêtres se passaient de siècle en siècle le savoir et les rites (p. 23). Mais apprendre, pour Claude Baillif, c'est aussi voir, ce qui le pousse à parcourir l'Italie, car les choses que l'on voit contiennent une âme (p. 42). Au retour toutefois, il ne réussit plus à retrouver Catherine, dont l'évocation du nom, du souvenir, remontera à la mémoire de Baillif chaque fois qu'une situation le provoquera: Fantôme de moi, de nous, illusion, chimère. (p. 165) Ainsi se poursuit le roman, sous les signes liés de la connaissance et de la souffrance.

Monseigneur de Laval demande cependant à Claude Baillif s'il consent à un engagement en Nouvelle-France. Baillif finira par 
accepter et sera l'architecte de nombreuses réalisations: Notre-Dame de Québec, Notre-Dame de la Victoire, la maison de Joliet, entre autres. Son chef-d'œuvre sera le palais épiscopal de Monseigneur de Saint-Vallier, encore qu'il gardera sa plus grande tendresse pour un moulin à eau plus utile mais, surtout, moins solennel. La Chair de pierre se clôt donc par les réalisations de Claude Baillif; mais encore ici apparaissent l'amour et la mort, puisque Baillif se trouvera quelques instants en présence de Catherine, sans toutefois jamais la reconnaître, et que lui-même mourra dans des circonstances floues.

À première vue, la Chair de pierre pourrait sembler relever d'une forme de biographie historique, malgré le rapport ambigu que le récit entretient avec l'histoire. En effet, le narrateur, dans une sorte de préliminaire, expose les limites de son entreprise de restitution, limites qui tiennent au peu de renseignements dont on dispose sur Claude Baillif, de même qu'au mensonge que représente toute reconstruction historique. Raconter devient-il alors impossible? Non, bien au contraire: l'invention pallie le manque de renseignements et la reconstitution historique n'hésite pas à s'affirmer comme récit: seule la fiction ne ment pas. (p. 9)

Dès lors, si jamais la Chair de pierre tient de la biographie historique, c'est d'une manière très libre; mais la richesse du roman repose avant tout sur un registre de discours qui, comme une portée musicale, en articulent les divers étagements. En effet, la Chair de pierre atteint sa plénitude artistique en superposant, du début à la fin, les discours artistique, philosophique et amoureux; voilà pourquoi un regard sur chacun de ceux-ci nous permettra maintenant d'étaler leurs résonances respectives et de mieux comprendre ainsi la cohérence de l'œuvre tout entière.

Le discours sur l'art, et en particulier sur l'architecture, circule évidemment tout au long du roman, mais il est particulièrement émouvant lorsque le jeune Claude Baillif s'initie à la pratique de son maître. Il s'y transmet alors un sens du mystère et du sacré qui commande un respect empreint d'humilité de l'être devant la connaissance. Quand Baillif pénètre les premiers secrets de l'architecture, c'est manifestement avec une attitude où l'être est au service de la connaissance, car celle-ci est le fruit d'un long mûrissement historique. Litinéraire de Claude Baillif en est d'abord un de maîtrise: il recopie les dessins du maître italien Palladio, il apprend tout par cœur, car le cœur y est véritablement. Toutefois, l'enseignement essentiel que Maître Nicolas de Limbourg lui donne, c'est qu'il faut posséder la connaissance avant de penser soi-même à la transformer:

Peut-être un jour deviendras-tu architecte et pourras-tu sortir des préceptes de Signor Andrea qui sont les meilleurs du monde, mais pour sortir d'un lieu faut-il d'abord y être entré 
puis le connaitre pour bien en trouver les issues. Ce sont des livres sacrés. Tu les remettras bien couchés, sur ces tavelettes de bouleau et tu veilleras chaque jour qu'ils soient essuyés de la poussière. (p. 24-25)

Cette attitude amène par ailleurs Claude Baillif à parcourir l'Italie justement parce que, d'abord, il faut voir: je reviendrai avec la connaissance, dit-il à Catherine avant de la quitter (p. 30). Ainsi, à propos de Galileo' Galilei, Baillif sait qu'il doit refaire le parcours de cet homme: Il me semble que je comprendrai bien cet homme que j'aime, celui qui chercha la vérité par constatation [...] si j'ai la chance de voir de mes yeux les lieux où il a vécu et qui ont gardé enfermées quelques parcelles de son esprit... (p. 42) Beaucoup plus tard, lorsqu'il sera rendu en Nouvelle-France; Baillif rencontrera Louis Joliet. Ce dernier lui expliquera son intense appétit de tout voir: Je suis ainsi, je ne tiens pas en place dans les séminaires et sur les quais, me faut une barque, et souquer, comme disent les Portugais. Je m'emplis les yeux. (p. 159) Claude Baillif découvre en cet homme un frère en ce que l'un et l'autre veulent cheminer vers la lumière: Il reconnut un autre feu plus familier, plus profond, celui de la connaissance, et lentement les deux hommes devinrent amis. (p.159)

Mais la connaissance de'son art, l'architecture, n'est pas pour Baillif une possession solitaire; au contraire; elle doit s'adresser au plus grand nombre. Tout au long de sa vie, Baillif se méfiera de l'art réservé aux riches, aux puissants, et cette réflexion orientera toute son activité: $\grave{A}$ quoi servait l'amour de connaître si la sagesse acquise ne donnait rien à ceux qui en méritaient le plus? (p. 59) Ainsi, construire des bâtiments de plus en plus beaux pour le clergé, c'était détourner l'édifice de ceux pour lesquels il fut conçu, c'était insulter la pauvreté (p. 149).

De même, Baillif se méfiera d'une certaine conception de l'art qui s'éloigne de l'ordre, de la simplicité, du silence. Profondément cartésien en cette matière - et le roman est d'ailleurs parsemé de nombreuses citations de Descartes - l'architecte se souviendra de cette parole de Claude François: Songe, petit Claude, que la meilleure prière est celle où le mot Dieu, seul, sürgit du silence, et que l'architecture ne parle qu'à voix basse. (p. 32) La vérité de l'architecture repose dans cette raison qu'a chaque pièce de l'ensemble d'être posée là où elle est, témoignant de la vision de son maître d'œuvre.

Le discours sur l'art s'avère en outre inséparable du discours philosophique, car l'architecture est, jusqu'à un certain point, un art du temps. Folch-Ribas fait jaillir ici et là dans son œuvre des réflexions qui montrent bien la nature ambiguë du rapport de l'architecture avec la durée. L'œuvre donne-t-elle l'impression de pérennité ? Alors ce serait illusion, car le temps la détruit. Mais que restet-il qui puisse survivre à la durée ? L'âme n'est pas dans les objets, elle est toute dans l'invention, et dans la façon. Tout ce qui peut se 
transmettre à ceux qui viendront après nous, ce sont les manières que nous avions. De nos constructions, bientôt, il ne reste plus rien. (p. 182) Baillif rappelle ensuite cette sentence des Pédauques: Sitôt la maison achevée, la mort y entre.

Et comme il y aurait beaucoup à dire sur le rapport qu'entretiennent l'architecture et la nature! Là aussi se présente un discours sur l'homme et son environnement qu'il faudrait méditer, tout comme celui qu'engendre chez Baillif la rencontre avec les "Sauvages", reposant tout entière la question de la connaissance qu'avait formulée Descartes: si la maîtrise et la possession de la nature devraient nous permettre de jouir de ses fruits, alors les Sauvages avaient-ils donc trouvé ces connaissances, car il jouissaient ainsi? (p. 104)

Enfin, un discours amoureux encadre tout le roman dans la personne de Catherine principalement, disparue tôt mais jamais oubliée. Baillif évóque son nom avec une régularité, une obsession incantatoire. Repensant à la mort de ses parents, de ses frères, Claude Baillif ressent un mal profond: Je portais en moi une grande blessure divisée en plusieurs cicatrices [...] et la dernière encore ouverte sous le lard et l'huile que j'y mettais: Catherine. (p. 55) Mais c'est aussi une perception du corps de la femme, en l'occurrence de Victoire, qui nous montre la grande unité de tous ces discours:

La chemise de nuit s'enroulait autour de Victoire en plis désordonnés, elle s'était froissée durant le sommeil. Claude tendait les mains et parcourait longuement la sculpture d'un corps qu'il découvrait sans éprouver le désir de la dévoiler. Il pensait que la vue de sa nudité le décevrait, tout comme celle de sa nature à lui, déplairait à Victoire. Ils ne parlèrent de cela ni l'un ni l'autre. Claude apprenait chaque fois cette architecture profonde comme s'il ne l'avait jamais encore touchée, jouissant du plaisir des surfaces qui se lient, des lignes qui se contredisent, des volumes qui se fondent, des odeurs sitôt nées qu'évanouies et des chaleurs paresseuses. (p. 137)

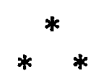

Renonçant au titre accrocheur, j'ai simplement (et clairement) dit ce que je pensais du roman de Folch-Ribas: exceptionnel. Rarement nous a-t-il été donné de lire, je crois, une œuvre aussi riche portée par une écriture manifestant une telle simplicité, un tel dépouillement. Vers la fin de sa vie, Baillif dit à Gédéon: Parler peu, c'est l'art; en quelques traits, tout dire... (p. 208). Jacque's Folch-Ribas le possède ce talent qui consiste à se diriger directement vers l'essentiel en peu de mots, par des phrases qui lancent la raison, la rêverie vers d'autres horizons, sans jamais se détacher du récit qui les porte. 
Il se dégage en outre, de la Chair de pierre, une profonde humilité face à la connaissance dont on ne peut tirer que de bénéfiques enseignements. Le cheminement de Baillif est exemplaire en ce qu'il s'inscrit dans la durée, dans l'histoire, l'être humain n'étant qu'une pierre d'un édifice qui se construit. En définitive, voilà un roman profondément religieux, c'est-à-dire qui relie, par un sens authentique du sacré, par un respect infini pour le passé et une passion pour le présent, tous les aspects de la vie d'un homme qui pourrait bien être un héros pour notre temps.

1. Jacques Folch-Ribas, la Chair de pierre, Paris, Robert Laffont, 1989, 234 p. (p. 22). 